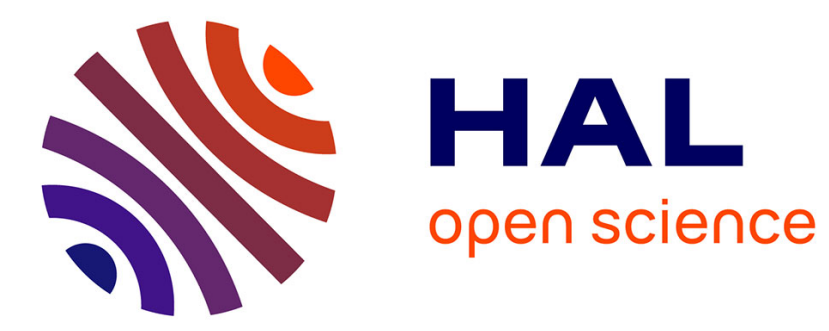

\title{
Two models of nonsmooth dynamical systems
}

Madeleine Pascal

\section{To cite this version:}

Madeleine Pascal. Two models of nonsmooth dynamical systems. International journal of bifurcation and chaos in applied sciences and engineering , 2011, 21 (10), pp.2853-2860. 10.1142/S0218127411030180 . hal-00653630

\section{HAL Id: hal-00653630 \\ https://hal.science/hal-00653630}

Submitted on 10 Jun 2016

HAL is a multi-disciplinary open access archive for the deposit and dissemination of scientific research documents, whether they are published or not. The documents may come from teaching and research institutions in France or abroad, or from public or private research centers.
L'archive ouverte pluridisciplinaire HAL, est destinée au dépôt et à la diffusion de documents scientifiques de niveau recherche, publiés ou non, émanant des établissements d'enseignement et de recherche français ou étrangers, des laboratoires publics ou privés. 


\title{
TWO MODELS OF NONSMOOTH DYNAMICAL SYSTEMS
}

\author{
MADELEINE PASCAL \\ Laboratoire IBISC, \\ Universite d'Evry Val d'Essonne, \\ 40 rue du Pelvoux, \\ 91020, Evry cedex, France \\ madeleine.pascal@ibisc.univ-evry.fr
}

\begin{abstract}
Two examples of nonsmooth systems are considered. The first one is a two degrees of freedom oscillator in the presence of a stop. A discontinuity appears when the system position reaches a critical value. The second example consists of coupled oscillators excited by dry friction. In this case, the discontinuity occurs when the system's velocities take a critical value. For both examples, the dynamical system can be partitioned into different configurations limited by a set of boundaries. Within each configuration, the dynamical model is linear and the close form solution is known. Periodic orbits, including several transitions between the various configurations of the system, are found in analytical form. The stability of these orbits is investigated by using the Poincaré map modeling.
\end{abstract}

Keywords: Systems with impact; dry friction oscillators; analytical investigation.

\section{Introduction}

Nonsmooth dynamical systems are related to force or motion characteristics which are noncontinuous. Nonsmooth phenomena are caused by kinematics constraints or physical effects like friction, impacts or backlash. For example, vibrating systems with clearance between the moving parts are frequently encountered in technical applications: intermittent contacts between the rotor and the stator of a turbo machine, due to unbalance, induce some undesirable effects. In many industrial applications like brake systems, machine tools, or wheel/rail contacts, dry friction leads to several serious problems. Nonsmooth systems are strongly nonlinear and they are usually modeled as spring mass oscillators. Several investigations consider the simple case of a one-degree of freedom system [Shaw \& Holmes, 1983; Himmarsh \& Jeffries, 1984; Andreus \& Casini, 2001; Liu \& Chang, 2002]; the case of multidegrees of freedom systems [Awrejcewicz, 1991; Aidanpan \& Gupta, 1993; Awrejcewicz et al., 2005], was up to now only investigated with a numerical approach. In this work, two examples of nonsmooth systems, including two degrees of freedom, are considered. Periodic orbits are found by using analytical methods.

\section{A Model of Systems with Clearance: Coupled Oscillators in the Presence of a Stop}

The system under consideration is composed of two masses $m_{1}$ and $m_{2}$ connected by linear springs of stiffness $k_{1}$ and $k_{2}$ (Fig. 1). The nonlinearity comes from the presence of a stop. When the displacement $x_{1}$ of the first mass is greater than the clearance, a contact of the mass with the stop occurs. This contact gives rise to a restoring force associated to a spring stiffness $k_{3}$. Two cases are considered. The first one is obtained when the stop stiffness goes to infinity (rigid impact). The second case is concerned by soft impact, with a finite value of $k_{3}$. 


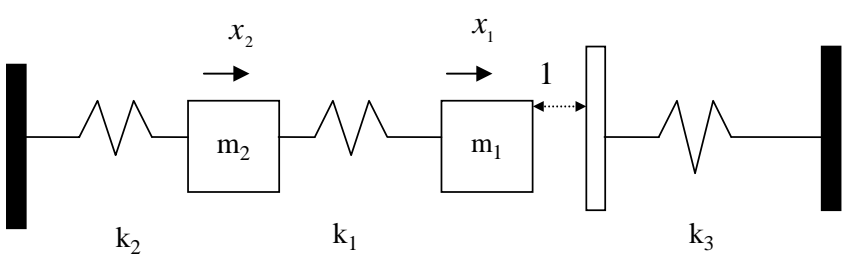

Fig. 1. Coupled oscillators with a stop.

\subsection{Rigid impact}

The corresponding dynamical model is given by:

$$
\begin{gathered}
M \ddot{x}+K x=0, \quad x=\left(\begin{array}{l}
x_{1} \\
x_{2}
\end{array}\right) \quad \text { if } x_{1}<1 \\
M=\left(\begin{array}{cc}
m_{1} & 0 \\
0 & m_{2}
\end{array}\right), \quad K=\left(\begin{array}{cc}
k_{1} & -k_{1} \\
-k_{1} & k_{1}+k_{2}
\end{array}\right) .
\end{gathered}
$$

If a contact of the first mass with the stop occurs $\left(x_{1}=1\right)$, the velocity of $m_{1}$ reverses according to Newton's impact law:

$$
x_{1}^{+}=-x_{1}^{-} \quad \text { (perfect elastic impact) }
$$

The system is a piecewise linear system limited by the boundary $x_{1}=1$. The analytical solution of Eq. (1) is given by:

$$
\begin{gathered}
X=H(t) X_{0}, \quad X=\left(\begin{array}{l}
x \\
\dot{x}
\end{array}\right), \quad x=\left(\begin{array}{l}
x_{1} \\
x_{2}
\end{array}\right), \\
X_{0}=X(0), \quad H(t)=\left(\begin{array}{ll}
H_{1} & H_{2} \\
H_{3} & H_{1}
\end{array}\right) .
\end{gathered}
$$

The 2 by 2 matrices $H_{i}(t),(i=1,2,3)$ are obtained [Pascal, 2006] from a modal analysis of the system (1).

\subsection{Soft impact}

The dynamical model is a piecewise linear system with two configurations limited by the boundary $x_{1}=1$. A first configuration (free motion) is obtained when the displacement of the first mass is smaller than the clearance $\left(x_{1}<1\right)$. The corresponding dynamical model is given by Eq. (1). A second configuration (constraint motion) occurs when $x_{1}>1$. The dynamical model in this case is given by:

$$
\begin{gathered}
M \ddot{x}+K_{1} x=K_{3}, \quad K_{3}=\left(\begin{array}{c}
k_{3} \\
0
\end{array}\right) \\
K_{1}=\left(\begin{array}{cc}
k_{1}+k_{3} & -k_{1} \\
-k_{1} & k_{1}+k_{3}
\end{array}\right)
\end{gathered}
$$

For a free motion, the related solution is given by Eq. (3). For a constraint motion, the corresponding solution is

$$
Z(t)=\tilde{H}(t) Z_{0}, \quad Z=\left(\begin{array}{c}
x-d_{0} \\
\dot{x}
\end{array}\right), \quad Z_{0}=Z(0)
$$

$d_{0}={ }^{t}\left(d_{01}, d_{02}\right)$ (constant part of the solution), $\tilde{H}(t)=\left(\begin{array}{cc}\tilde{H}_{1}(t) & \tilde{H}_{2}(t) \\ \tilde{H}_{3}(t) & \tilde{H}_{1}(t)\end{array}\right)$ is obtained [Pascal, 2006] from a modal analysis of the constraint system (4).

\subsection{Periodic motions (rigid impact)}

A family of periodic solutions including one rigid impact per period is obtained in analytical form [Pascal, 2006]. The corresponding initial conditions: $X_{0}=\left(\begin{array}{l}x_{0} \\ \dot{x}_{0}\end{array}\right), x_{0}=\left(\begin{array}{l}1 \\ y\end{array}\right), \dot{x}_{0}=\left(\begin{array}{l}u \\ w\end{array}\right)$ are defined in terms of the period $T$ and the initial velocity $w$ of the nonimpacting mass is zero.

\subsection{Periodic motions (soft impact)}

A set of periodic orbits including one constraint phase $(0<t<\tau)$ and one free phase $\left(\tau<t<\tau+\tau_{1}\right)$ per period is obtained in analytical form [Pascal, 2006]. The time duration of each part of the motion

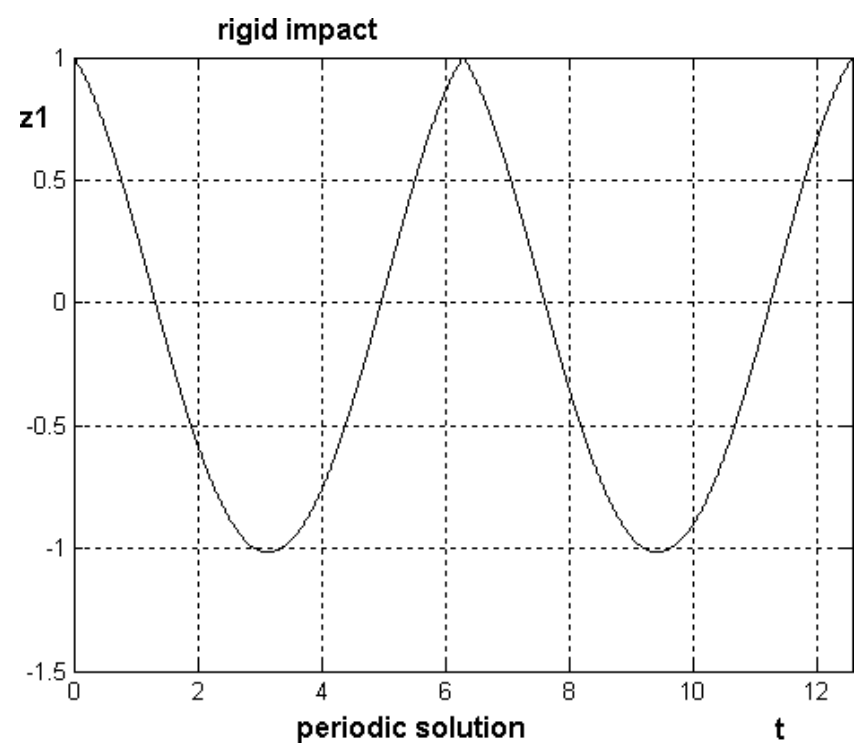

Fig. 2. Time history of the first mass. 


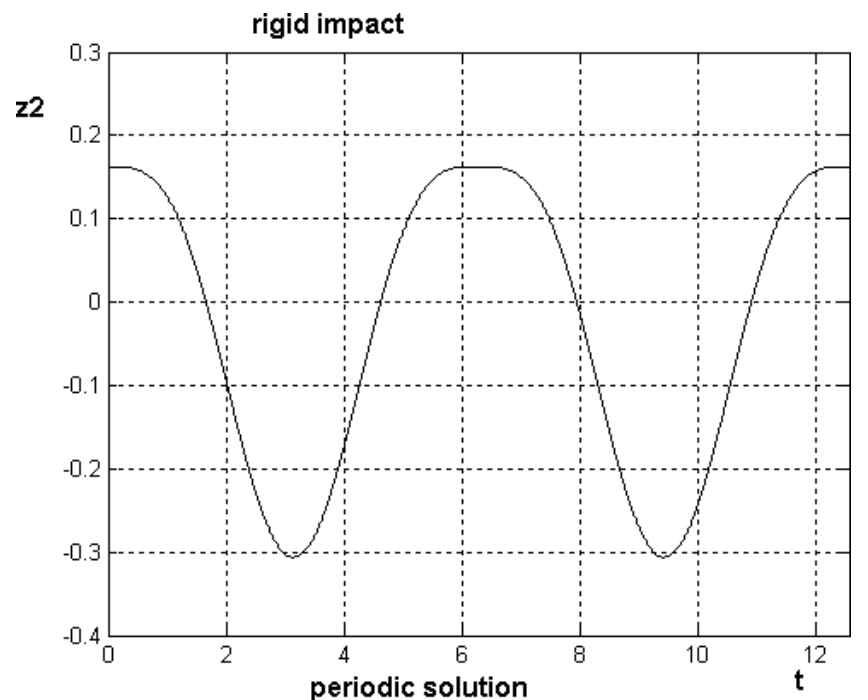

Fig. 3. Time history of the second mass.

is computed. At the end of the constraint phase, the positions and the velocities of the system are obtained by the rule: $X(\tau)=\left(\begin{array}{c}x_{0} \\ -\dot{x}_{0}\end{array}\right)$.

The corresponding initial conditions $X_{0}=$ $\left(\begin{array}{l}x_{0} \\ \dot{x}_{0}\end{array}\right), x_{0}=\left(\begin{array}{l}1 \\ y\end{array}\right), \dot{x}_{0}=\left(\begin{array}{l}u \\ w\end{array}\right)$ are obtained in terms of the period $T=\tau+\tau_{1}$. These periodic orbits tend to the periodic orbits related to rigid impact when the stop's stiffness tends to infinity.

The numerical validation of these analytical results is performed in [Pascal, 2006]. In the case of rigid impact, the time history of the masses (during two periods) is shown in Figs. 2 and 3, for the

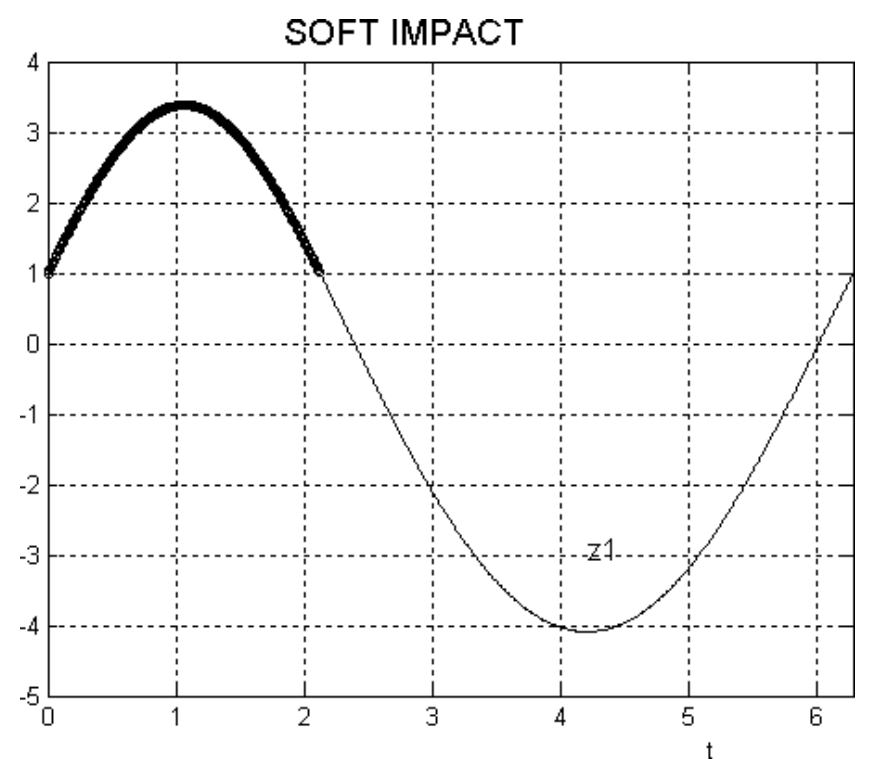

Fig. 4. Time history of the first mass.

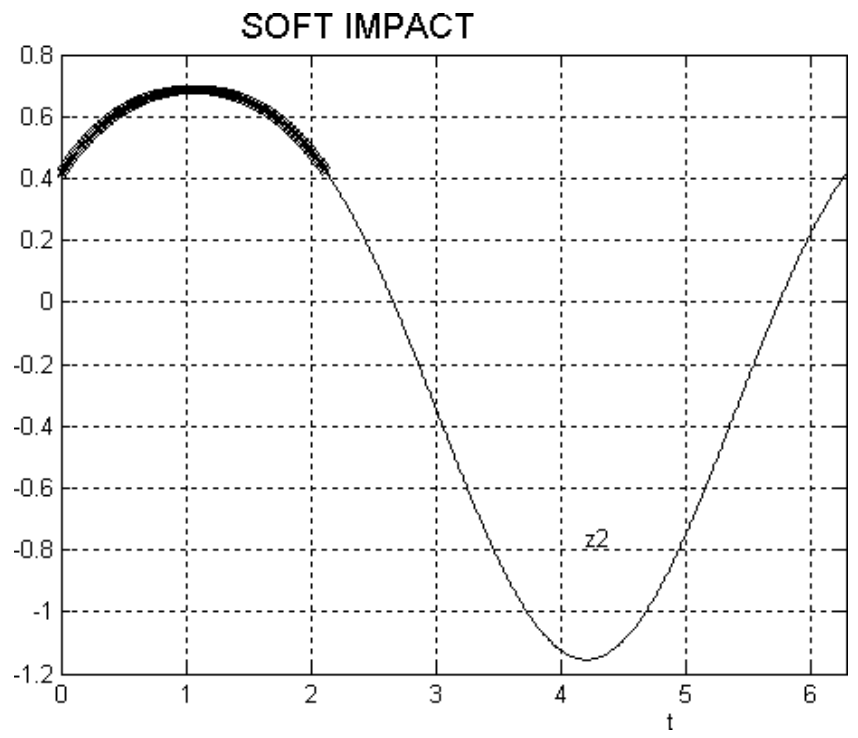

Fig. 5. Time history of the second mass.

following values of the data:

$$
m_{1}=1, \quad m_{2}=2, \quad k_{1}=1, \quad k_{2}=5, \quad T=2 \pi
$$

In the case of soft impact, the time history of the system (during one period) is shown in Figs. 4 and 5 for a stop's stiffness $k_{3}=1$ and the same values of the other parameters.

\section{Coupled Oscillators Excited by Dry Friction}

The system (Fig. 6) consists of two masses $m_{1}, m_{2}$ connected by linear springs of stiffness $k_{1}, k_{2}$. These two masses are in contact with a driving belt moving at a constant velocity $V$. Friction forces $F_{1}, F_{2}$ act between the masses and the belt.

The motion equations are written in the following form:

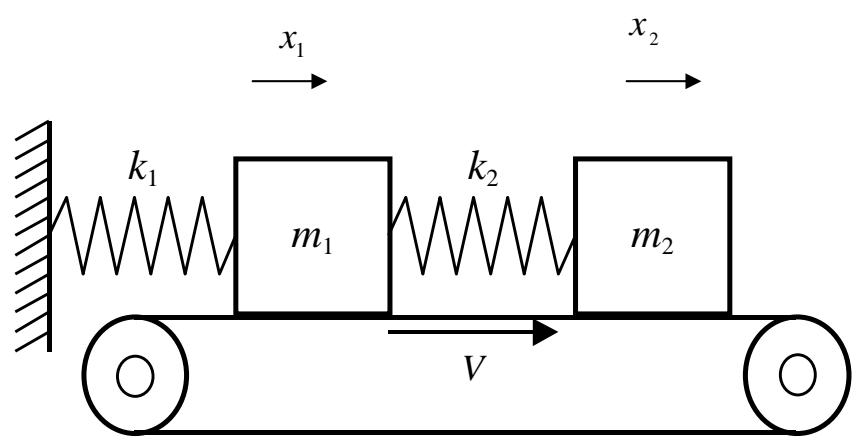

Fig. 6. Two degrees of freedom oscillator with dry friction. 


$$
\begin{gathered}
x_{1}^{\prime \prime}+x_{1}-\chi x_{2}=u_{1}, \quad \chi=\frac{k_{2}}{k_{1}+k_{2}}, \quad \eta=\frac{m_{1}}{m_{2}} \\
x_{2}^{\prime \prime}+\chi \eta\left(x_{2}-x_{1}\right)=\eta u_{2}, \quad u_{i}=\frac{F_{i}}{k_{1}+k_{2}} \quad(i=1,2) \\
t=\omega_{3} t^{\prime}, \quad \omega_{3}=\sqrt{\frac{k_{1}+k_{2}}{m_{1}}}, \quad\left(o^{\prime}\right)=\frac{d(o)}{d t^{\prime}}
\end{gathered}
$$

The friction forces are obtained from Coulomb's laws:

- $V-x_{i}^{\prime} \neq 0, u_{i}=u_{s i} \operatorname{sign}\left(V-x_{i}^{\prime}\right)(i=1,2)(\operatorname{slip}$ motion of $m_{1}, m_{2}$ )

- $V-x_{1}^{\prime}=0$

$$
u_{1}=\left\{\begin{array}{cc}
x_{1}-\chi x_{2} & \text { if }\left|x_{1}-\chi x_{2}\right|<u_{r 1} \\
\varepsilon u_{s 1} & \left(\text { stick motion of } m_{1}\right) \\
& \text { if } \varepsilon\left(x_{1}-\chi x_{2}\right)>u_{r 1}(\varepsilon= \pm 1) \\
& \left(\text { slip motion of } m_{1}\right)
\end{array}\right.
$$

- $V-x_{2}^{\prime}=0$

$$
u_{2}=\left\{\begin{array}{lc}
\chi\left(x_{2}-x_{1}\right) & \text { if }\left|\chi\left(x_{2}-x_{1}\right)\right|<u_{r 2} \\
& \left(\text { stick motion of } m_{2}\right) \\
\varepsilon u_{s 2} & \text { if } \varepsilon \chi\left(x_{2}-x_{1}\right)>u_{r 2}(\varepsilon= \pm 1) \\
& \left(\text { slip motion of } m_{2}\right)
\end{array}\right.
$$

$u_{s i}<u_{r i} \quad(i=1,2)$

$u_{s i}$ is the slipping friction force, while $u_{r i}$ is the static friction force.

\subsection{Prediction of the oscillations exhibited by the system}

The dynamical behavior of this piecewise linear system is very complex and includes slip or stick oscillations of the masses. All possible motions are composed of several phases of slip motion for the both masses, stick motion of $m_{1}$ and slip motion of $m_{2}$ or vice versa, and finally, stick motion for the two masses. For each kind of motions, the close form solution is obtained [Pascal, 2008].

Global Slipping Phase: The motion equations are written as:

$$
\begin{gathered}
z^{\prime \prime}+\tilde{K} z=0, \quad \tilde{K}=\left(\begin{array}{cc}
1 & -\chi \\
-\chi \eta & \chi \eta
\end{array}\right) \\
z=\left(z_{1}, z_{2}\right)^{t}, \\
z_{i}=x_{i}-\tilde{d}_{0 i}, \quad z_{i}^{\prime}<V, \quad(i=1,2) \\
\tilde{d}_{01}=\frac{u_{s 1}+u_{s 2}}{1-\chi}, \quad \tilde{d}_{02}=\frac{\chi u_{s 1}+u_{s 2}}{\chi(1-\chi)}
\end{gathered}
$$

The solution is given by:

$$
Z(t)=h(t) Z_{0}, \quad Z=\left(\begin{array}{c}
z \\
z^{\prime}
\end{array}\right), \quad Z_{0}=Z(0)
$$

The 4 by 4 matrix $h(t)$ [Pascal, 2008] is deduced from a modal analysis of system (6).

Slip-Stick Motion:

$$
\begin{gathered}
z^{\prime \prime}+\tilde{K}_{1} z=0, \quad \tilde{K}_{1}=\left(\begin{array}{cc}
1 & -\chi \\
0 & 0
\end{array}\right) \\
z_{1}^{\prime}<V, \quad z_{2}^{\prime}=V \\
e_{2}-2 u_{r 2}<\chi\left(z_{2}-z_{1}\right)<e_{2} \\
\left(e_{2}=u_{r 2}-u_{s 2}\right)
\end{gathered}
$$

The solution is: $Z(t)=\Gamma(t) Z_{0}$, where the 4 by 4 matrix $\Gamma(t)$ results from a modal analysis of system (7).

\section{Stick-Slip Motion:}

$$
\begin{gathered}
z^{\prime \prime}+\tilde{K}_{2} z=0, \quad \tilde{K}_{2}=\left(\begin{array}{cc}
0 & 0 \\
-\chi \eta & \chi \eta
\end{array}\right) \\
z_{1}^{\prime}=V, z_{2}^{\prime}<V \\
e_{1}-2 u_{r 1}<z_{1}-\chi z_{2}<e_{1} \\
\left(e_{1}=u_{r 1}-u_{s 1}\right)
\end{gathered}
$$

The solution [Pascal, 2008] is given by a 4 by 4 matrix $C(t)$ related to a modal analysis of system (8).

$$
Z(t)=C(t) Z_{0}
$$

Global Stick Motion: The motion equations in this case are:

$$
\begin{gathered}
z^{\prime \prime}=0, \quad z_{1}^{\prime}=z_{2}^{\prime}=V \\
e_{1}-2 u_{r 1}<z_{10}-\chi z_{20}<e_{1} \\
e_{2}-2 u_{r 2}<\chi\left(z_{20}-z_{10}\right)<e_{2}
\end{gathered}
$$


The solution is given by:

$Z(t)=D(t) Z_{0}, \quad D=\left(\begin{array}{cc}I & V t I \\ 0 & I\end{array}\right), \quad I=\left(\begin{array}{ll}1 & 0 \\ 0 & 1\end{array}\right)$.

A transition from one kind of motion to another occurs if some switching conditions are fulfilled. One of the mass switches from slip to stick motion, if at some instant of time, its velocity reaches the velocity of the belt and if the restoring force exerted on this mass is smaller than the related static friction force. On the other hand, a transition from stick to slip motion occurs at some instant of time, if the restoring force applied to the mass reaches the limiting value (i.e. static friction force). In the following, two sets of periodic solutions including several transitions per period are obtained in analytical form.

\subsection{Symmetrical periodic solution}

A first set of periodic motions, including two transitions per period, is obtained.

Starting from the initial conditions:

$$
x_{10}^{\prime}<V, \quad x_{20}^{\prime}=V, \quad \chi\left(x_{20}-x_{10}\right)=u_{r 2}
$$

the first part of the motion $(0<t<\tau)$ is a global slip motion, and the second part $\left(\tau<t<\tau+\tau_{1}\right)$ is a slip motion for the first mass and a stick motion for the second one. At the end of the global slip motion, the system positions and velocities are obtained by the rule:

$$
z(\tau)=-z_{0}, \quad z^{\prime}(\tau)=z_{0}^{\prime}
$$

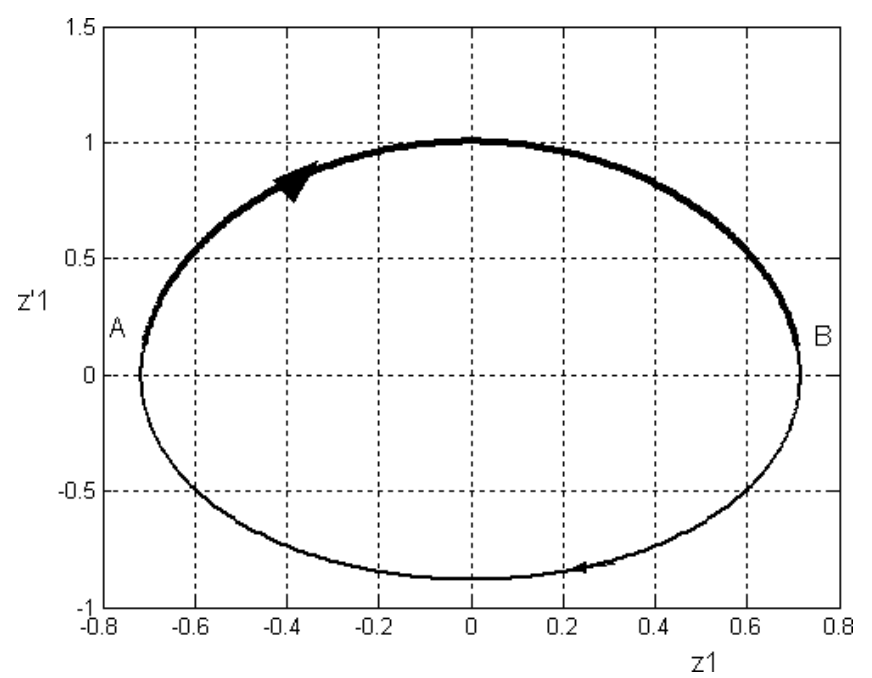

Fig. 7. Phase portrait of the first mass.

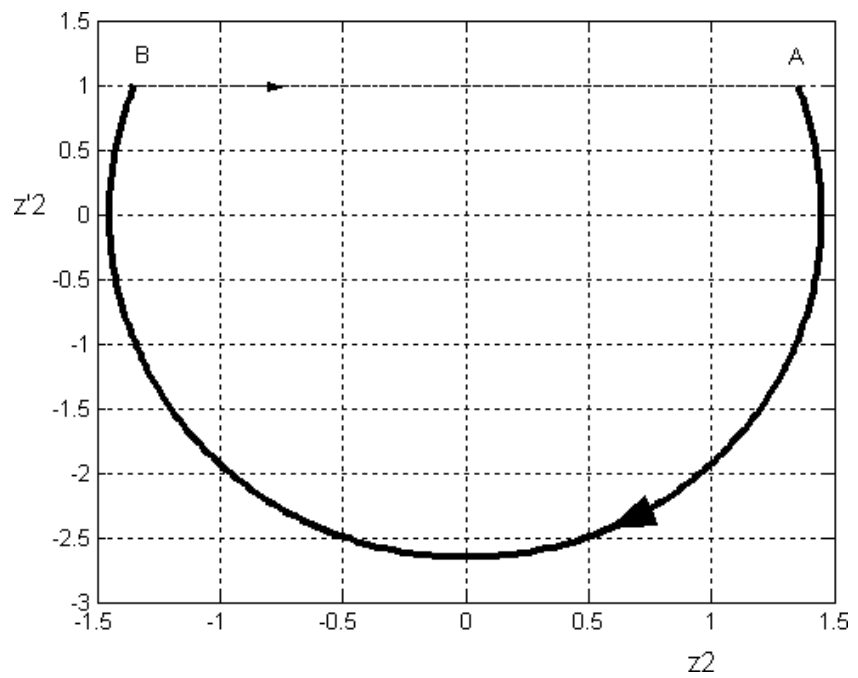

Fig. 8. Phase portrait of the second mass.

A detailed formulation of the numerical validation of these results can be found in [Pascal, 2008].

For the following values of the data:

$$
\begin{gathered}
\chi=0.4, \quad \eta=4, \quad V=0.6773, \quad u_{s 1}=0.0127, \\
u_{r 1}=0.0255, \quad u_{s 2}=0.5, \quad u_{r 2}=1
\end{gathered}
$$

we obtain:

$$
\tau=2.65, \quad \tau_{1}=2.2407
$$

Moreover, the phase portraits of the system (Figs. 7 and 8) are symmetrical with respect to the lines corresponding to the constant part of the solution.

\subsection{Periodic solutions including a global sticking phase}

A new kind of periodic motions including a global sticking phase and four transitions per period is also obtained in analytical form. For each period, starting from initial conditions

$$
\begin{gathered}
x_{10}^{\prime}=x_{20}^{\prime}=V, \\
\left|x_{10}-\chi x_{20}\right|<u_{r 1}, \quad \chi\left|x_{20}-x_{10}\right|<u_{r 2}
\end{gathered}
$$

the solution involves a global stick motion given by Eq. (10) for $0<t<t_{a}$.

This motion ends when a restoring force applied to the masses reaches the limiting value $u_{r i}$. For all the motion, the restoring force applied to the second mass is constant $\left(z_{2}-z_{1}=z_{20}-z_{10}\right)$. A transition occurs at $t=t_{a}$, when the restoring force applied to the first mass reaches his limiting value. Let us 
define:

$$
\begin{gathered}
Z_{a} \equiv Z\left(t_{a}\right)=D\left(t_{a}\right) Z_{0}, \quad Z_{a}=\left(\begin{array}{c}
z_{a} \\
z_{a}^{\prime}
\end{array}\right) \\
z_{a}^{\prime}=(V, V)^{t}, \quad z_{a}=\left(z_{1 a}, z_{2 a}\right)^{t}, \quad z_{1 a}-\chi z_{2 a}=e_{1}
\end{gathered}
$$

For a new period of time: $t_{a}<t<t_{a}+\tau, M_{1}$ performs a slip motion, while $M_{2}$ is still in stick motion:

$$
Z(t)=\Gamma\left(t-t_{a}\right) Z_{a}
$$

This motion ends at $t=t_{a}+\tau$, when the restoring force applied to the second mass reaches its limiting value. Let us define:

$$
\begin{gathered}
Z_{c} \equiv Z\left(t_{a}+\tau\right)=\Gamma(\tau) Z_{a}, \quad Z_{c}=\left(\begin{array}{c}
z_{c} \\
z_{c}^{\prime}
\end{array}\right) \\
z_{c}^{\prime}=\left(z_{1 c}^{\prime}, V\right)^{t}, \quad z_{c}=\left(z_{1 c}, z_{2 c}\right)^{t} \\
\chi\left(z_{2 c}-z_{1 c}\right)=e_{2}
\end{gathered}
$$

A new phase occurs for $t_{a}+\tau<t<t_{a}+$ $\tau+\tau_{1}$, related to a global slip motion of the system, defined by:

$$
Z(t)=H\left(t-t_{a}-\tau\right) Z_{c}
$$

This motion ends at $t=t_{a}+\tau+\tau_{1}$, if at that time:

$$
\begin{gathered}
z_{1 d}^{\prime} \equiv z_{1}^{\prime}\left(t_{a}+\tau+\tau_{1}\right)=V, \\
e_{1}-2 u_{r 1}<z_{1 d}-\chi z_{2 d}<e_{1} \\
z_{i d}=z_{i}\left(t_{a}+\tau+\tau_{1}\right), \quad(i=1,2)
\end{gathered}
$$

For $t_{a}+\tau+\tau_{1}<t<t_{a}+\tau+\tau_{1}+\tau_{2}$, the first mass performs a stick motion $\left(z_{1}^{\prime}=V\right)$, while the second one performs a slip motion:

$$
\begin{aligned}
Z(t) & =C\left(t-t_{a}-\tau-\tau_{1}\right) Z_{d}, \\
Z_{d} & =Z\left(t_{a}+\tau+\tau_{1}\right)
\end{aligned}
$$

A periodic motion of period $t_{a}+\tau+\tau_{1}+\tau_{2}$ is obtained if:

$$
Z\left(t_{a}+\tau+\tau_{1}+\tau_{2}\right)=Z_{0}
$$

The obtained periodic motion depends on six parameters: $z_{10}, z_{20}, t_{a}, \tau, \tau_{1}, \tau_{2}$ and the switching conditions (12), (14), (16), together with the periodicity conditions (18) provides six scalar equations for the determination of these parameters. This new periodic solution is a generalization of the solution II obtained in [Pascal, 2008]: the solution II is a

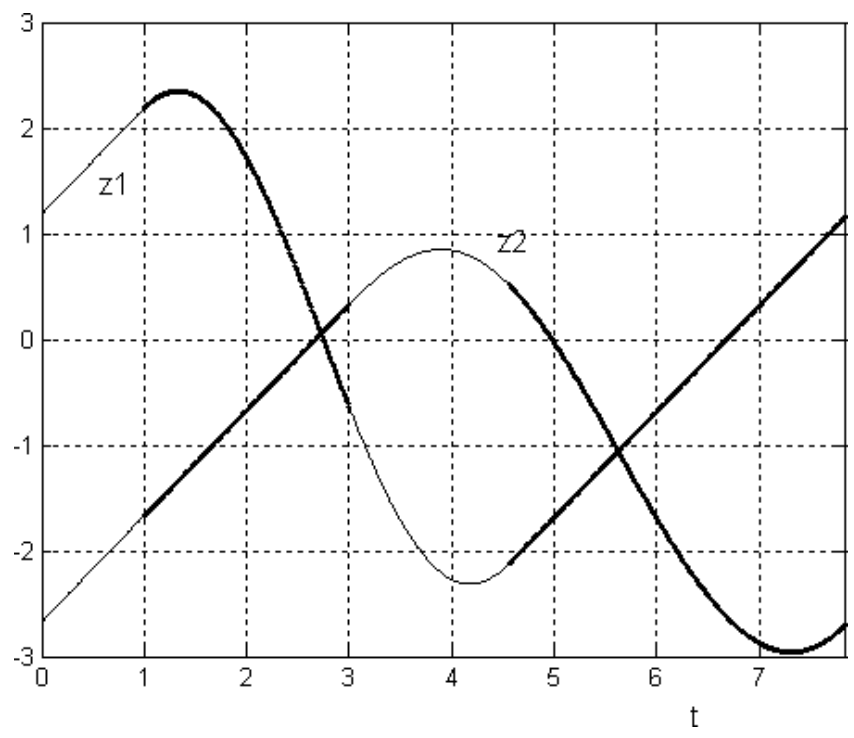

Fig. 9. Time history of the system.

particular case of this new solution corresponding to $t_{a}=0$. A detailed formulation of the numerical validation of these analytical results are given in the appendix.

For the following values of the data:

$\chi=0.5, \quad \eta=V=1, \quad e_{1}=3.0195, \quad e_{2}=0.4831$, we obtain

$$
\begin{gathered}
t_{a}=1, \quad \tau=2, \quad \tau_{1}=1.568, \quad \tau_{2}=3.28, \\
z_{10}=1.1838, \quad z_{20}=-2.6715 .
\end{gathered}
$$

The corresponding behavior of the system is shown in Figs. 9 and 10.

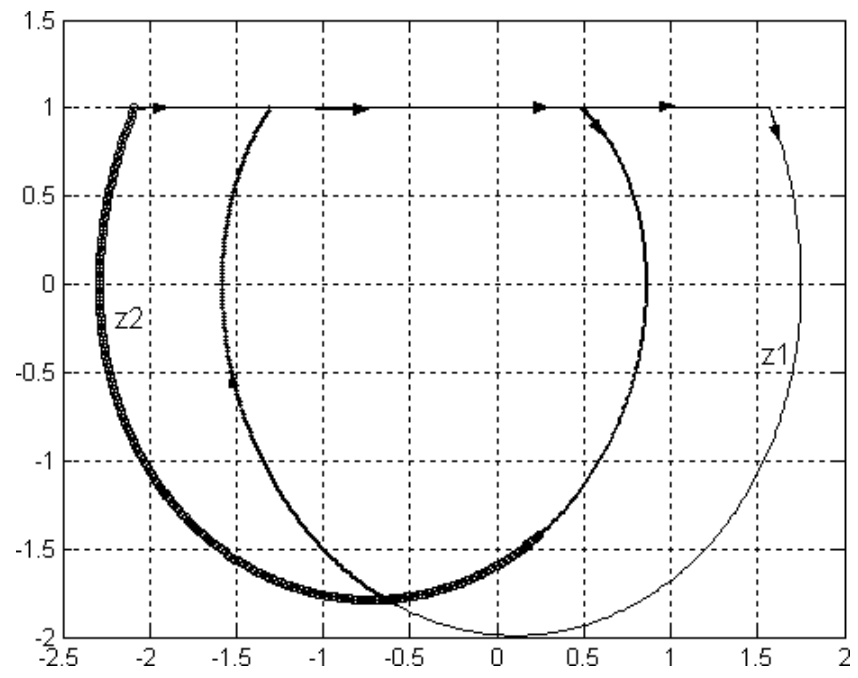

Fig. 10. Phase plot of the system. 


\section{Stability of the Periodic Solutions for Nonsmooth Systems}

For nonsmooth systems, due to the presence of discontinuities in the motion equations, the stability of the periodic solutions cannot be investigated by the classical linearization method. An alternative way [Natsiavas, 1998] consists of using Poincaré map modeling. The phase space is partitioned into different configurations $\left(\Phi_{1}\right), \ldots,\left(\Phi_{n}\right)$ limited by several boundaries $\left(\Sigma_{0}\right),\left(\Sigma_{1}\right), \ldots,\left(\Sigma_{n}\right)$. At the boundary $\left(\Sigma_{k}\right)$ between two adjacent configurations (Fig. 11), the system switches from one configuration to another. The time $t_{k}$ of switching is obtained from the corresponding switching condition. Within each configuration $\left(\Phi_{k}\right)$, the solution $\varphi_{k}(t)$ is known. For a periodic orbit, the final switching surface $\left(\Sigma_{n}\right)$ is identical to $\left(\Sigma_{0}\right)$. A Poincaré map from $\left(\Sigma_{0}\right)$ to $\left(\Sigma_{0}\right)$ is defined by $P=P_{n} \circ \cdots \circ P_{k} \circ \cdots \circ P_{1}$, where $P_{k}$ is a local mapping between $\left(\Sigma_{k-1}\right)$ and $\left(\Sigma_{k}\right)$ defined by:

$$
Z\left(t_{k}\right)=\varphi_{k}\left(t_{k}-t_{k-1}\right) Z\left(t_{k-1}\right)
$$

The fixed points of the map define the periodic solutions.

The stability of one fixed point of the map depends on the eigenvalues of the Jacobian matrix $J$ of the map. If one (at least) of these eigenvalues lies (in the complex plan) outside of the unit circle, the fixed point of the map is unstable, and the corresponding periodic orbit is also unstable. If all the eigenvalues of $J$ are inside the unit circle, the fixed point of the map is stable, but the related periodic solution is only conditionally stable, with respect to some particular perturbations of the initial conditions leading to the same kinds of switches that occur in the unperturbed motion.

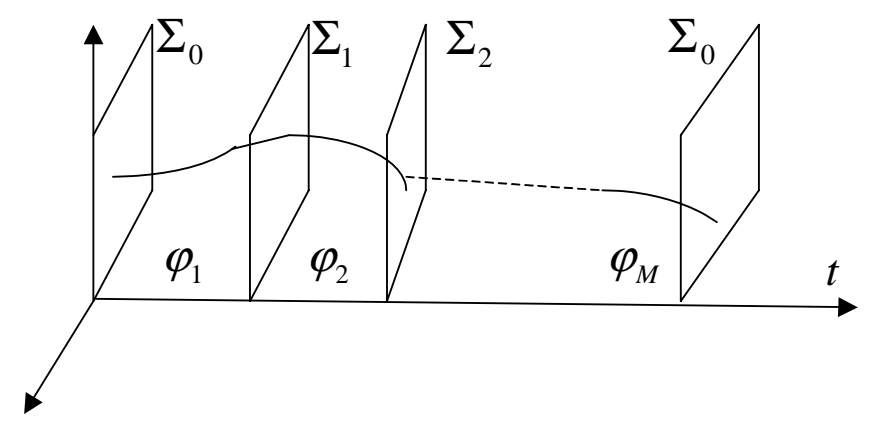

Fig. 11. Poincaré map.

\section{Application of the Poincaré Map Modeling: Stability of Periodic Solutions in the Case of Dry Friction Oscillators}

An example of the investigation of the stability of periodic solutions for nonsmooth systems is presented in the following. We consider the periodic orbit including a global stick phase, which was obtained in the case of the coupled oscillators with dry friction. This solution involves four configurations (global stick motion, slip-stick motion, global slip motion, stick-slip one), limited by five boundaries:

$$
\begin{aligned}
& \left(\Sigma_{0}\right): x_{1}^{\prime}=x_{2}^{\prime}=V \\
& \left(\Sigma_{1}\right): x_{1}^{\prime}=x_{2}^{\prime}=V, \quad x_{1}-\chi x_{2}=u_{r 1} \\
& \left(\Sigma_{2}\right): x_{2}^{\prime}=V, \quad \chi\left(x_{2}-x_{1}\right)=u_{r 2} \\
& \left(\Sigma_{3}\right): x_{1}^{\prime}=V, \\
& \left(\Sigma_{4}\right) \equiv\left(\Sigma_{0}\right)
\end{aligned}
$$

The stability of the periodic solution is determined by the eigenvalues of the matrix $J$ given the correspondence between the initial perturbations in $\left(\Sigma_{0}\right): d Z_{0}={ }^{t}(x, y, 0,0)$ and the final ones (also in $\left.\left(\Sigma_{0}\right): d Z_{f}={ }^{t}\left(x_{f}, y_{f}, 0,0\right)\right)$ :

$$
\left(\begin{array}{l}
x_{f} \\
y_{f}
\end{array}\right)=J\left(\begin{array}{l}
x \\
y
\end{array}\right)
$$

Due to the degeneracy of the phase space during the global stick motion (in this case, the phase space dimension is only two instead of four during global slip motion), the Jacobian matrix $J$ is a 2 by 2 singular matrix and the periodic solution is conditionally stable if $|\operatorname{trace}(J)|<1$. Several other results on the stability of the periodic solutions obtained in the examples of nonsmooth systems considered in this paper can be found in [Pascal, 2006, 2008].

\section{Concluding Remarks}

A unified approach for two models of nonsmooth systems is considered in this paper. The dynamical behavior of nonlinear oscillators in the presence of discontinuities of forces or of motions is investigated. For systems with clearance, the discontinuities appear when the system's position reaches some critical value. For systems with dry friction, a discontinuity holds when the velocities reach a 
critical value. The corresponding dynamical model is strongly nonlinear. However, the system can be partitioned into different configurations. Within each configuration, the dynamical model is linear and the closed form solution of the motion equations is available. It results that, even in the case of multidegrees of freedom systems, it is possible to obtain the solution of these nonlinear systems by patching the solutions obtained in the successive configurations. Several periodic solutions are obtained in analytical form for a two degrees of freedom systems with clearance or in the presence of dry friction. The stability of these particular solutions is also investigated. This paper extends the results obtained by the author in two previous papers.

\section{References}

Aidanpan, J. O. \& Gupta, R. D. [1993] "Periodic and chaotic behavior of a threshold-limited two degree of freedom system," J. Sound Vib. 165, 305-327.

Andreaus, U. \& Casini, P. [2001] "Dynamics of friction oscillators excited by a moving base and/or driving force," J. Sound Vib. 245, 685-699.

Awrejcewicz, J. [1991] Bifurcation and Chaos in Coupled Oscillators (World Scientific, Singapore).

Awrejcewicz, J., Dzyubak, J. \& Grebogi, C. [2005] "Estimation of chaotic and regular (stick-slip and slip-slip) oscillations exhibited by coupled oscillators with dry friction," Nonlin. Dyn. 42, 383-394.

Hinmarsh, M. B. \& Jeffries, D. J. [1984] "On the motions of the impact oscillator," J. Phys. A 17, 1791-1803.

Liu, C. S. \& Chang, W. T. [2002] "Frictional behavior of a belt-driven and periodic excited oscillator," J. Sound Vib. 258, 247-268.

Natsiavas, S. [1998] "Stability of piecewise linear oscillators with viscous and dry friction damping," J. Sound Vib. 217, 507-522.

Pascal, M. [2006] "Dynamics and stability of a two degrees of freedom oscillator with an elastic stop," ASME J. Comput. Nonlin. Dyn. 1, 94-102.

Pascal, M. [2008] "Dynamics of coupled oscillators excited by dry friction," ASME J. Comput. Nonlin. Dyn. 3, 20-26.
Shaw, S. W. \& Holmes, P. J. [1983] "A periodically forced piecewise linear oscillator," J. Sound Vib. 90, 129-155.

\section{Appendix}

The periodicity conditions (18) give the following equations:

$$
\begin{aligned}
& \left(\tilde{N}_{1}-I\right) z_{0}+\tilde{N}_{2} z_{0}^{\prime}=0 \\
& \tilde{N}_{3} z_{0}+\left(\tilde{N}_{4}-I\right) z_{0}^{\prime}=0 \\
& \tilde{N}=C(T) H\left(\tau_{1}\right) \Gamma(\tau) D\left(t_{a}\right)=N D\left(t_{a}\right) \\
& N=\left(\begin{array}{ll}
N_{1} & N_{2} \\
N_{2} & N_{4}
\end{array}\right), \quad \tilde{N}=\left(\begin{array}{cc}
\tilde{N}_{1} & \tilde{N}_{2} \\
\tilde{N}_{2} & \tilde{N}_{4}
\end{array}\right) \\
& z_{0}^{\prime}=(V, V)^{t}
\end{aligned}
$$

These periodicity conditions include the last switching condition (16).

From (A.1), we deduce two compatibility conditions

$$
\begin{gathered}
\tilde{M}_{11}+\tilde{M}_{12}=0 \\
\tilde{M}_{21}+\tilde{M}_{22}=0 \\
\tilde{M}=\left(\tilde{M}_{i j}\right)_{i, j=1.2} \\
\tilde{M}=\left(\tilde{N}_{1}-I\right)^{-1} \tilde{N}_{2}-\left(\tilde{N}_{3}\right)^{-1}\left(\tilde{N}_{4}-I\right)
\end{gathered}
$$

The relation (A.2) provides two equations liking the time duration $\left(t_{a}, \tau, \tau_{1}, T\right)$ of each parts of the motion; from (A.1), we deduce the value of $z_{0}$

$$
z_{0}=-\left(\tilde{N}_{1}-I\right)^{-1} \tilde{N}_{2} z_{0}^{\prime}
$$

Inserting this result in the switching conditions (12) and (14), we obtain two new relations for the determination of the parameters $t_{a}, \tau, \tau_{1}, T$. Several conditions about the results obtained by this numerical method must be fulfilled: for example, the computed values of the time duration $t_{a}, \tau, \tau_{1}, T$ must be positive numbers. 